\title{
Probing a Kondo-Correlated Quantum Dot with Spin Spectroscopy
}

\author{
D. Kupidura, ${ }^{1}$ M. C. Rogge, ${ }^{1, *}$ M. Reinwald, ${ }^{2}$ W. Wegscheider, ${ }^{2}$ and R. J. Haug ${ }^{1}$ \\ ${ }^{1}$ Institut für Festkörperphysik, Universität Hannover, Appelstraße 2, D-30167 Hannover, Germany \\ ${ }^{2}$ Angewandte und Experimentelle Physik, Universität Regensburg, D-93040 Regensburg, Germany
}

(Received 14 September 2005; published 1 February 2006)

\begin{abstract}
We investigate the Kondo effect and spin blockade observed in a many-electron quantum dot and study the magnetic field dependence. At lower fields, a pronounced Kondo effect is found, which is replaced by the spin blockade at higher fields. In an intermediate regime, both effects are visible. We make use of this combined effect to gain information about the internal spin configuration of our quantum dot. We find that the data cannot be explained assuming regular filling of electronic orbitals. Instead, spin polarized filling seems to be probable.
\end{abstract}

DOI: 10.1103/PhysRevLett.96.046802

Quantum dot systems containing confined electrons have been studied intensively both theoretically [1] and experimentally [2] during the past decades. They have proven to be excellent systems to explore the physics of confined charge carriers. Beyond the Coulomb blockade $[1,3]$, many effects have been found to reveal quantum mechanical and spin properties of confined electrons, among them the Kondo effect and spin blockade. The Kondo effect is caused by correlated cotunneling events [4,5] resulting in enhanced conductance when sequential tunneling is Coulomb blocked. The underlying mechanism is due to the formation of a spin singlet between conduction electrons and the localized spin in the quantum dot. Spin blockade as introduced by Ciorga et al. [6] appears in lateral dot systems when applying a magnetic field perpendicular to the sample surface. Spin dependent transport suppression is found as a consequence of electron injection from spin polarized edge states.

In this Letter, we investigate the interplay between these two effects, as they appear in the same sample in slightly different magnetic field regions. We study the magnetoconductance for the Kondo effect and spin blockade individually and the transition between them. Results from both effects are then combined to gain information about the internal spin configuration of the quantum dot.

Our device is defined by terms of local anodic oxidation [7] on a GaAs/AlGaAs heterostructure containing a 2dimensional electron system (2DES) $37 \mathrm{~nm}$ below the surface. The 2DES has an electron density of $n_{e}=3.94 \times$ $10^{15} \mathrm{~m}^{-2}$ and mobility of $\mu_{e}=52.7 \mathrm{~m}^{2} \mathrm{~V}^{-1} \mathrm{~s}^{-1}$ at $4.2 \mathrm{~K}$. Figure 1(a) shows an atomic force microscope (AFM) picture of our dot with leads source $(S)$ and drain $(D)$ being the electron reservoirs and in plane gates $\left(G_{1}-G_{4}\right)$, respectively. Gates $G_{1}$ and $G_{4}$ are designed to control the coupling to the leads, while gates $G_{2}$ and $G_{3}$ control the electrostatic potential of the dot. The measurements are performed in a $\mathrm{He}^{3} / \mathrm{He}^{4}$ dilution refrigerator at $50 \mathrm{mK}$ with a magnetic field $B$ applied perpendicular to the 2DES. Standard lock-in technique is used to record the differential
PACS numbers: 73.63.Kv, 72.15.Qm, 73.21.La, 73.23.Hk

conductance $G$ as a function of gate voltages and magnetic field.

Figure 1(b) shows a simplified model of our system in the relevant field regime. The properties of the dot are basically characterized by the two lowest Landau levels formed at the edge and the core of the dot (filling factor $\nu_{\text {dot }}>2$ ). The 2-dimensional leads are subject to the quantum Hall effect and, thus, develop spin polarized edge channels that get separated in space with increasing $B$. The visibility of the Kondo effect and spin blockade depends on this separation and, thus, on the strength of the magnetic field.

For relatively low fields up to approximately $3.5 \mathrm{~T}$, the leads can still be considered as unpolarized. Here we find Kondo physics. The Kondo effect is induced by a common state of electrons on the dot and electrons in the leads. If high tunnel coupling presumed - the total spin of the dot is $S=1 / 2$, a singlet state can be formed with the leads allowing cotunneling events even if sequential tunneling is Coulomb blocked. As a consequence, finite transport is observed between individual Coulomb peaks. Since transport via this Kondo state involves spin flips, both spins must be present in the leads.
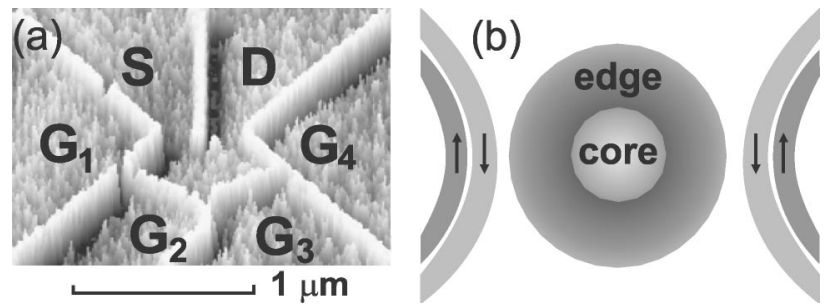

FIG. 1. (a) AFM picture of our device with source $(S)$ and drain $(D)$ leads and gates $\left(G_{1}\right.$ to $\left.G_{4}\right)$. (b) Schematic picture: Under the influence of a magnetic field, the dot can be represented as edge and core areas for the two lowest Landau levels. The dot is coupled to source and drain represented here by spin polarized edge states. 
Figure 2(a) shows a measurement in the relevant Kondo regime. The differential conductance $G$ is measured as a function of the voltage applied to gate 3 and the magnetic field. Several Coulomb peaks are visible. Their peak positions, e.g., the ground state energies, exhibit a zigzag behavior as the field increases. Negative gradients correspond to transport through the edge of the dot, while transport through the center leads to a positive gradient. In between, we find a regular pattern of enhanced conductance caused by a Kondo effect involving the edge of the dot. The center of the dot is not involved in this Kondo effect, since the tunnel coupling to the leads is not high enough. Thus, transport via a Kondo singlet state corresponds to a total spin $S=1 / 2$ at the edge of the dot. This spin depends on the number of electrons at the edge, which can be changed in two different ways. An electron can enter the edge either coming from the leads or coming from the dot center. The first mechanism is induced by increasing the gate voltage increasing the total number of electrons. Therefore, a regular modulation of the Kondo effect is found as a function of $V_{G 3}$. This is demonstrated in Fig. 2(b), where $G$ is plotted as a function of $V_{G 3}$ along the dashed line in Fig. 2(a). The alternating Kondo effect is visible as an alternating height of successive Coulomb valleys. The second mechanism is used when increasing the magnetic field. With increasing $B$, electrons are redis-
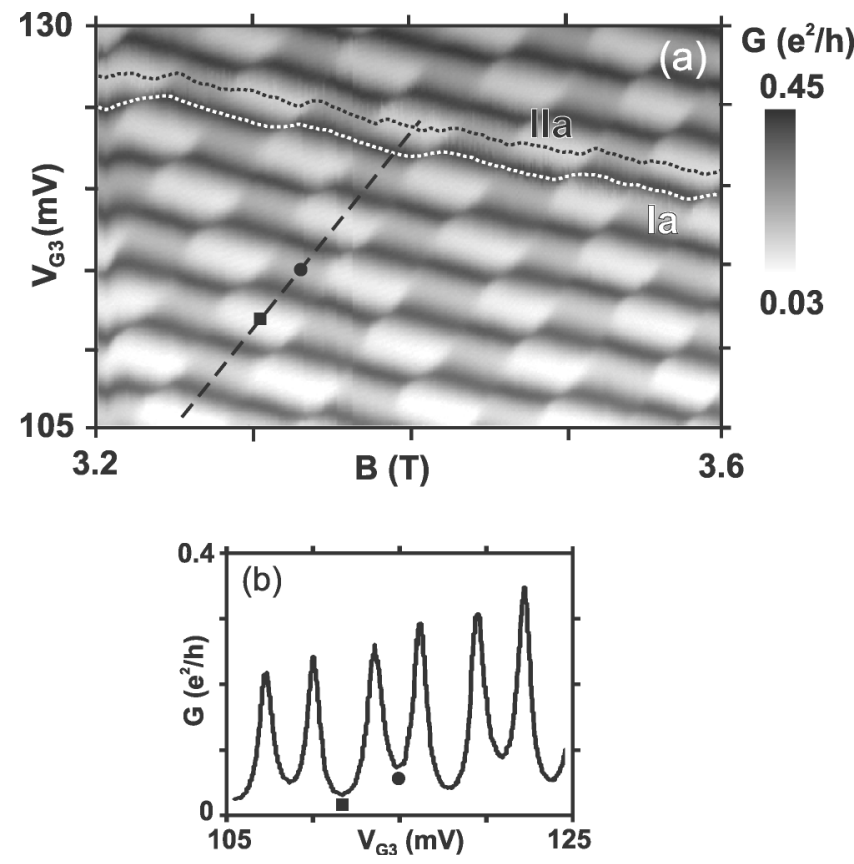

FIG. 2. (a) Differential conductance $G$ as a function of magnetic field $B$ and gate voltage $V_{G 3}$. A chessboard pattern is observed caused by Kondo conductance in the Coulomb valleys between individual Coulomb peaks. The Kondo effect is modulated by electron number and magnetic field. (b) A trace along the dashed line in (a) demonstrates the alternating height of successive Coulomb valleys (exemplarily marked with a square and a circle). tributed from the second Landau level in the center to the first Landau level at the edge. This leads to a Kondo modulation as a function of $B$ and overall to a chessboard pattern as reported in Refs. [8-11].

Above $3.5 \mathrm{~T}$, the spatial separation of the edge channels becomes prominent, and the leads are more and more spin polarized leading to a pronounced spin blockade. This is visible in Fig. 3(a). Again, several Coulomb peaks are visible as a function of $V_{G 3}$ and $B$ but this time for a higher field range. The zigzag pattern of the peak position still reflects the energy of the ground states located at the center of the dot (positive slope) or at the edge (negative slope). In addition, a bimodal behavior of the peak amplitude is found when transport occurs through the edge. This is shown in Fig. 3(b). The peak amplitude for the peak marked as Ib in Fig. 3(a) is plotted as a function of $B$. Since the edge states in the leads are spatially separated, the overlap of the wave functions between the lead and the dot for the energetically higher spin state (spin up in the following) is reduced as the more distant edge state is involved and transport is blocked. In contrast, electrons with the lower spin state (spin down) coming from the closer edge state cause an unsuppressed transport. This effect allows one to detect the spin of an electron added to the dot. Crossing a Coulomb peak in the gate voltage direction with an unsuppressed amplitude leads to the
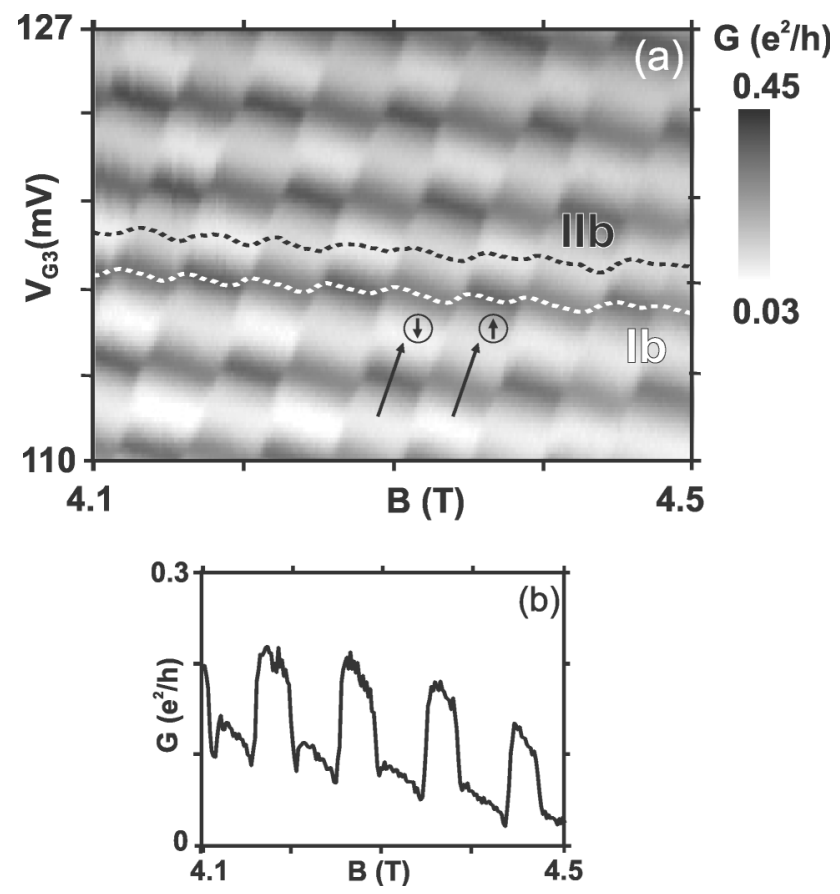

FIG. 3. (a) At higher fields, spin polarized edge states in the leads give rise to the spin blockade. Transport with spin up electrons is suppressed compared to spin down transport. The arrows denote charging with the assigned spin detectable with the amplitude of the corresponding Coulomb peak. (b) Peak amplitude for peak Ib. The bimodal behavior due to spin blockade is clearly visible. 
addition of an electron with spin down, while the addition via a low amplitude corresponds to a spin up electron [see arrows in Fig. 3(a)].

So far, both regimes were investigated separately. In the following, we will concentrate on the interplay between the Kondo effect and spin blockade. The peak amplitudes of the peaks marked as Ia and Ib in Figs. 2 and 3 are plotted in gray as a function of magnetic field in Fig. 4. The strength of the bimodal behavior reflects the strength of the spin blockade. At 3.2 T, almost no oscillation is visible. The leads are still unpolarized, and, thus, no spin blockade is observed. With increasing $B$, the edge states start to split and the spin blockade becomes visible. Above $4 \mathrm{~T}$, it reaches its maximum. The black curve represents the conductance in the Coulomb valley (IIa and IIb in Figs. 2 and 3 ) above the depicted peak. At $3.2 \mathrm{~T}$, a strong oscillation is observed due to a pronounced Kondo effect. Increasing the field separates the edge states. This leads to a suppression of the Kondo effect, since the transport over the Kondo state involves spin flips requiring both spin orientations in the leads. At 4.5 T, the Kondo effect is strongly suppressed.

In an intermediate regime around $4 \mathrm{~T}$, both effects are visible simultaneously. Thus, the results from both effects can be combined. We find that a high peak amplitude corresponding to spin down transport is accompanied by a suppressed Kondo effect in the Coulomb valley above. Therefore, the addition of an electron to the energetically lower spin down state does not lead to a total spin $S=1 / 2$ required for Kondo conductance. Instead, the Kondo effect reflecting a total spin of $S=1 / 2$ appears above a low peak amplitude when an electron with the energetically higher spin up state is added. This discrepancy shows that the electronic state structure within our dot cannot be described in terms of noninteracting electrons. A simple

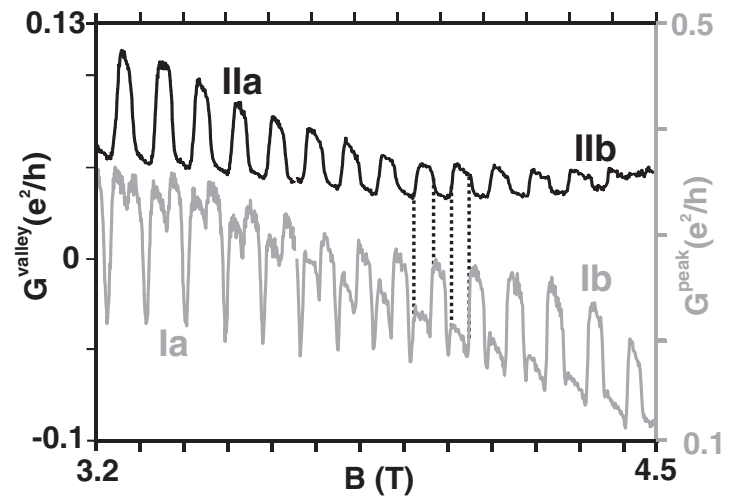

FIG. 4. Peak amplitude along Coulomb peak Ia/Ib (gray curve) and conductance in the Coulomb valley IIa/IIb above (black curve). While the bimodal behavior along the peak increases with magnetic field due to spin blockade, the oscillations due to the Kondo effect in the valley vanish. Around $4 \mathrm{~T}$, both effects are visible and comparable. A high amplitude at the peak goes along with a suppressed conductance in the valley (see dotted lines). model with regular filling of dot orbitals featuring a spin singlet phase is not applicable. Instead, the addition of electrons is realized favoring spin polarized configurations, i.e., a sort of Hund's rule.

We propose an explanation based on theoretical results inspired by an experiment given by Ciorga et al. [12]. They investigated the $\nu_{\mathrm{dot}}=2$ transition in a lateral quantum dot featuring spin blockade as well but no Kondo effect. A phase transition from a spin singlet phase towards a spin polarized phase was found depending on the number of electrons. For electron numbers below approximately 30, the spin singlet phase was observed with a regular filling of dot orbitals. Above this critical number, spin polarized configurations were observed.

We find that the model developed by Ciorga et al. for the internal spin configuration above the critical electron number can be applied to our results which fits the assumption of having a high electron number $N \approx 160$. The model is shown in Fig. 5. Four "Kondo fields" are shown with Kondo conductance in the lower left and the upper right [Fig. 5(a) shows the measurement and Fig. 5(b) the model]. Thus, in these fields we assume the total spin of the edge to be $S=1 / 2$. This is explained with the highest orbital in the edge to be half filled with a spin down electron. Going from the lower left to the upper left, a Coulomb peak is crossed with a high amplitude. Thus, the half filled orbital is not filled with a spin up electron. Instead, the next orbital is entered with a spin down electron. The total spin of the edge is $S=1$, and the Kondo effect disappears. If now the magnetic field is increased, an electron is redistributed from the center to the edge and the first half filled orbital at the edge is filled, leaving one half filled orbital with the total spin $S=1 / 2$. The Kondo effect is restored.

We want to mention that the results obtained here may have a universal character. Very similar results were obtained by Keller et al. [9]. A Kondo induced chessboard pattern was observed. The peak amplitude which behaves
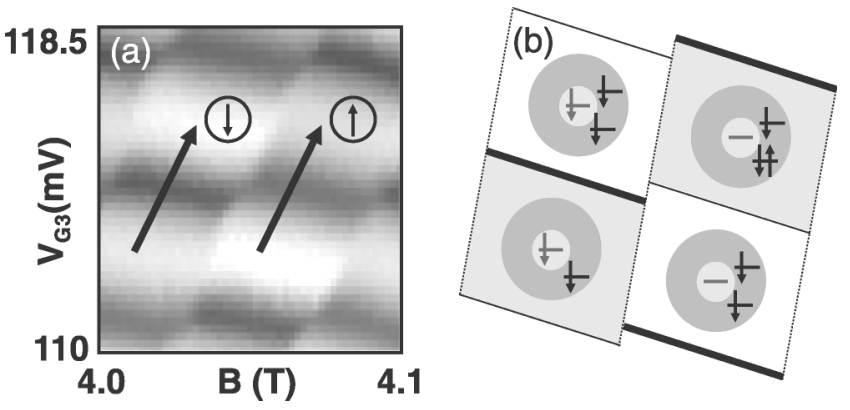

FIG. 5. (a) Increased region of Fig. 3(a) showing both the Kondo effect and spin blockade (same color scale as in Figs. 2 and 3). (b) Model of the spin configuration of the dot gained from the combination of results from the Kondo effect and spin blockade. The coincidence of low conductance along a peak and high conductance in the valley above is explained with spin polarized filling of electrons. 
the same way as in our sample was plotted, but no attention was paid to this effect. The chessboard was explained with regular filling featuring a spin singlet phase. This is, in our opinion, not valid. Instead, an electron number of approximately 50 supports our assumption of spin polarized filling. Measurements done by Fühner et al. [8] for an electron number of approximately 180 showing the magnetic flux dependence of the chessboard pattern also confirm our results. Other chessboard measurements combined with calculations were performed by Stopa et al. [10]. With an electron number below 30, they assumed a regular filling. Their calculations might, therefore, not be applicable for chessboard patterns at higher electron numbers. Regular filling was also obtained in Refs. [11,13]. The electron number in Ref. [13] is slightly above 50 but seems still to be low enough for the spin singlet phase probably due to different dot parameters.

In conclusion, we investigate the Kondo effect and spin blockade in a lateral quantum dot depending on the strength of a magnetic field applied perpendicular to the sample surface. At lower fields, we observe a Kondo induced chessboard pattern modulated by the number of electrons and the magnetic field. At higher fields, the leads become spin polarized and spin blockade sets in, making the electronic spin detectable. The Kondo conductance vanishes here, since both spin orientations in the leads are needed for the Kondo effect. In intermediate fields, both effects are observed. A combined analysis reveals the internal spin configuration of the dot. We find that the measurements cannot be described by a regular filling of electronic orbitals within a model of noninteracting electrons. Instead, for this high electron number a different approach featuring spin polarized filling with a sort of Hund's rule must be assumed. The comparison with other results challenges former interpretations of chessboard patterns and yields a consistent picture with the dot phase depending on the electron number.

This work has been supported by BMBF.

*Electronic address: rogge@ nano.uni-hannover.de

[1] C. W. J. Beenakker, Phys. Rev. B 44, 1646 (1991).

[2] L. P. Kouwenhoven, D. G. Austing, and S. Tarucha, Rep. Prog. Phys. 64, 701 (2001).

[3] M. A. Kastner, Phys. Today 46, No. 1, 24 (1993).

[4] S. Sasaki, S. De Franceschi, J. M. Elzerman, W. G. van der Wiel, M. Eto, S. Tarucha, and L. P. Kouwenhoven, Nature (London) 405, 764 (2000).

[5] D. Goldhaber-Gordon, Hadas Shtrikman, D. Mahalu, David Abusch-Magder, U. Meirav, and M. A. Kastner, Nature (London) 391, 156 (1998).

[6] M. Ciorga, A.S. Sachrajda, P. Hawrylak, C. Gould, P. Zawadzki, S. Jullian, Y. Feng, and Z. Wasilewski, Phys. Rev. B 61, R16315 (2000).

[7] M. Ishii, and K. Matsumoto, Jpn. J. Appl. Phys. 34, 1329 (1995).

[8] C. Fühner, U. F. Keyser, R. J. Haug, D. Reuter, and A. D. Wieck, Phys. Rev. B 66, 161305(R) (2002).

[9] M. Keller, U. Wilhelm, J. Schmid, J. Weis, K. von Klitzing, and K. Eberl, Phys. Rev. B 64, 033302 (2001).

[10] M. Stopa, W. G. van der Wiel, S. De Franceschi, S. Tarucha, and L. P. Kouwenhoven, Phys. Rev. Lett. 91, 046601 (2003).

[11] J. Schmid, J. Weis, K. Eberl, and K. v. Klitzing, Physica (Amsterdam) 9E, 54 (2001).

[12] M. Ciorga, A. Wensauer, M. Pioro-Ladriere, M. Korkusinski, J. Kyriakidis, A. S. Sachrajda, and P. Hawrylak, Phys. Rev. Lett. 88, 256804 (2002).

[13] M. C. Rogge, F. Cavaliere, M. Sassetti, R. J. Haug, and B. Kramer, cond-mat/0507036. 